\title{
On the discrete spectrum of the Dirac operator on bent chain quantum graph
}

\author{
Michail Belov ${ }^{1}$, Igor Popov ${ }^{1, *}$, Irina Blinova ${ }^{1}$ \\ ${ }^{1}$ ITMO University, Department of Higher Mathematics, 197101 St. Petersburg, Russia
}

\begin{abstract}
We study Dirac operators on an infinite quantum graph of a bent chain form which consists of identical rings connected at the touching points by $\delta$-couplings with a parameter $\alpha \in \mathbb{R}$. We are interested in the discrete spectrum of the corresponding Hamiltonian. It can be non-empty due to a local (geometrical perturbation of the corresponding infinite chain of rings. The quantum graph of analogous geometry with the Schrodinger operator on the edges was considered by Duclos, Exner and Turek in 2008. They showed that the absence of $\delta$-couplings at vertices (i.e. the Kirchhoff condition at the vertices) lead to the absence of eigenvalues. We consider the relativistic particle (the Dirac operator instead of the Schrodinger one) but the result is analogous. Quantum graphs of such type are suitable for description of grapheme-based nanostructures. It is established that the negativity of $\alpha$ is the necessary and sufficient condition for the existence of eigenvalues of the Dirac operator (i.e. the discrete spectrum of the Hamiltonian in this case is not empty). The continuous spectrum of the Hamiltonian for bent chain graph coincides with that for the corresponding straight infinite chain. Conditions for appearance of more than one eigenvalue are obtained. It is related to the bending angle. The investigation is based on the transfer-matrix approach. It allows one to reduce the problem to an algebraic task. $\delta$-couplings was introduced by the operator extensions theory method.
\end{abstract}

\section{Introduction}

Investigation of the physical properties of various compounds of half-crystals and nanotubes becomes more and more interesting. There are a lot of works dedicated to mathematical modeling of nanostructures, because it allows one to create diodes, transistors, amplifiers and more complex nano-scale devices (see, e.g., [1-4]). Many physical properties are related with the spectral characteristics of the system Hamiltonian. That is why the spectral problems attract additional attention. Hamiltonians of real physical systems are very complicated. Physicists usually used model Hamiltonians. Quantum graph is one of prospective models. It is simple and, at the same time, describes properties of physical systems $[5,6]$.

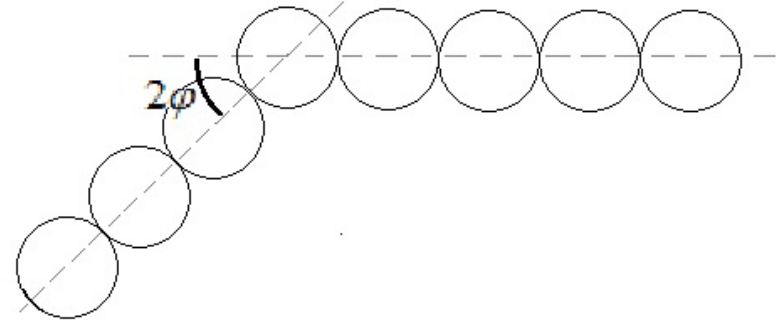

Fig. 1. A bent graph.
Mathematical models based on quantum graphs, as opposed to discrete, have a large number of parameters that allows one to make the model more realistic [7]. Usually, the Schrödinger operator is considered on the graph edges. It corresponds to non-relativistic quantum particle. As for a relativistic particle, i.e. the Dirac operator, the corresponding graph models are not so widely used. At present, due to the remarkable progress of graphene nanostructures (described better by the Dirac operator than by the Schrödinger one), the relativistic model found new applications.

In the present paper we consider bent chain graph (see Fig. 1) with the Dirac operator at the edges. A few years ago, the graph of the same geometry with the Schrödinger operator was studied. It was proved the existence of bound states for the case of delta-coupling at the graph vertices [8]. The analogous problems for branching graphs were studied in $[9,10]$. The mathematical background for introducing of the pointlike potentials is given by the theory of self-adjoint extensions of symmetric operators [11-13]. 1D point-like potential for the Dirac operator was described in many works (see, e.g., [14-21].

\section{Infinite periodic chain}

The Dirac operator in one dimension for spinless particle is described by the following expression (see, e.g., [11]):

\footnotetext{
* Corresponding author: popov1955@gmail.com
} 


$$
D_{0}=i c \frac{d}{d x} \otimes \sigma_{1}+\frac{c^{2}}{2} \otimes \sigma_{2},
$$

$\operatorname{Dom}\left(D_{0}\right)=H^{1}(R) \otimes C^{2}$, where $H^{1}(R)$ is the

Sobolev space $c, c>0$, is the speed of light $\sigma_{1}$ and $\sigma_{2}$ are the Pauli matrices,

$$
\sigma_{1}=\left(\begin{array}{ll}
0 & 1 \\
1 & 0
\end{array}\right), \sigma_{2}=\left(\begin{array}{cc}
1 & 0 \\
0 & -1
\end{array}\right),
$$

Consider bent chain graph $\Gamma$ shown in Fig. 1. It consists of two semi-infinite straight chains and a central region connecting them. At first, we will deal with the chains. Each chain is semi-periodic, i.e. it is invariant in respect to a semi-group of shifts by circular diameter. Each arc (edge of the graph) is parameterized by the polar angle $\phi$, the variable is $x=R \phi, R$ is the circle radius. For convenience, we take $R=1$ (it is not a limitation). One defines the Dirac operator $D$ on the graph by the following way. It acts in $L_{2}(\Gamma) \otimes C^{2}$ as $D_{0}$ at each edge. The operator domain consists of all 2vector-functions

$$
\left(\begin{array}{l}
\psi_{1}^{(\mathrm{j})}(x, \lambda) \\
\psi_{2}^{(\mathrm{j})}(x, \lambda)
\end{array}\right) \in H^{1}(\Gamma) \otimes C^{2}
$$

satisfying the following conditions at vertices (see Fig. 2):

$$
\left\{\begin{array}{l}
\sum_{j=1}^{4} \psi_{2}^{(\mathrm{j})}(0)=-\frac{i \alpha}{c} \psi_{1}(0), \\
\psi_{1}(0)=\psi_{1}^{(\mathrm{j})}(0), \quad \forall j=1, . ., 4 .
\end{array}\right.
$$

Here $-\frac{i \alpha}{c}$ characterizes the Lorentz interaction (relativistic point-like interaction).

If one consider the spectral problem, then it is necessary to solve the following Dirac system at each edge:

$$
D_{0} \psi=\lambda \psi
$$

Let $C_{1,2}^{(\mathrm{j})}$ be the value of function $\psi_{1,2}^{(\mathrm{j})}$ at the input ( $j=1,2)$ or the output ( $j=3,4)$ of the cell (Fig. 2). Given constants $C_{1,2}^{(1)}, C_{1,2}^{(2)}$ determines the solution at the whole cell and, correspondingly, the values $C_{1,2}^{(3)}, C_{1,2}^{(4)}$. Matrix $T$ which gives one this relation is named the transfer-matrix (or monodromy matrix) [22]. This relation gives one:

$$
\left(\begin{array}{l}
C_{1}^{(2 \mathrm{n}-1)} \\
C_{2}^{(2 \mathrm{n}-1)} \\
C_{1}^{(2 \mathrm{n})} \\
C_{2}^{(2 \mathrm{n})}
\end{array}\right)=T^{n}\left(\begin{array}{l}
C_{1}^{(1)} \\
C_{2}^{(1)} \\
C_{1}^{(2)} \\
C_{2}^{(2)}
\end{array}\right) .
$$

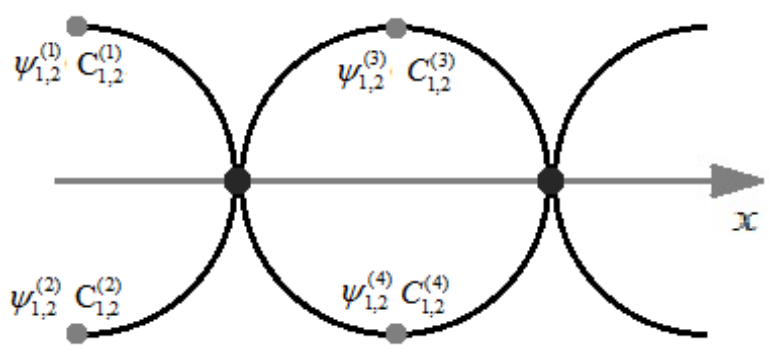

Fig. 2. The transfer-matrix definition.

To construct the transfer-matrix, one chooses directions at each edge of the basic cell (Fig. 3).

Let $\quad \psi_{1,2}^{(1)}(\mathrm{x}, \lambda), \quad \psi_{1,2}^{(2)}(\mathrm{x}, \lambda), \quad \psi_{1,2}^{(3)}(\mathrm{x}, \lambda) \quad$ and $\psi_{1,2}^{(4)}(\mathrm{x}, \lambda)$ be the solutions of the Dirac system (2.1) for the corresponding edge $\left(x \in\left[0, \frac{\pi}{2}\right]\right)$.

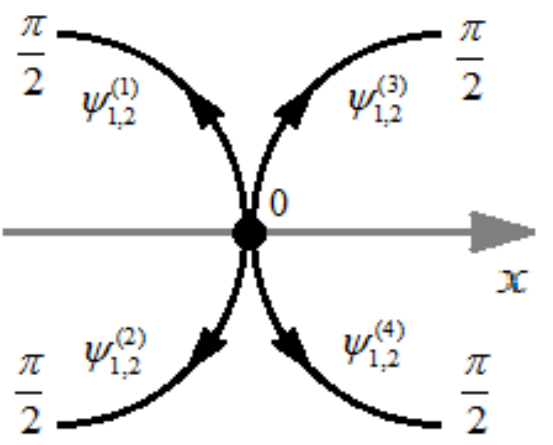

Fig. 3. The basic cell.

One starts from the given values $\psi_{1,2}^{(1)}\left(\frac{\pi}{2}, \lambda\right)=\mathrm{C}_{1,2}^{(1)}, \psi_{1,2}^{(2)}\left(\frac{\pi}{2}, \lambda\right)=\mathrm{C}_{1,2}^{(2)}$. The graph is symmetric. The condition at the vertex (2.0) is also symmetric. Hence, the solution should be symmetric or anti-symmetric at the cell. An anti-symmetric solution gives one zero values at the vertex, correspondingly, it is a trivial solution. We are interested in a symmetric case:

$$
\left\{\begin{array}{l}
\psi_{1}^{(1)}\left(\frac{\pi}{2}\right)=\psi_{1}^{(2)}\left(\frac{\pi}{2}\right) \\
\psi_{2}^{(1)}\left(\frac{\pi}{2}\right)=\psi_{2}^{(2)}\left(\frac{\pi}{2}\right)
\end{array} .\right.
$$

Taking into account (2.0) and (2.2), one can find the expression for the coefficients $C_{1,2}^{(3)}$ and $C_{1,2}^{(4)}$ through $C_{1,2}^{(1)}$ and $C_{1,2}^{(2)}$. Thus, one constructs the transfer-matrix.

\section{Main result}

Theorem. The Dirac operator $D$ for bent chain quantum graph has at least one bound state if and only if the parameter of the point-like potentials at the vertices is negative $(\alpha<0)$. 
Proof. Let's solve the Dirac system (2.1) at an edge. It is the homogeneous system of linear first order differential equations with constant coefficients. Its solutions can be written as:

$$
\left\{\begin{array}{l}
\psi_{1}=i \sqrt{\frac{c^{2}+2 \lambda}{c^{2}-2 \lambda}}\left(C_{1} e^{k x}-C_{2} e^{-k x}\right) \\
\psi_{2}=C_{1} e^{k x}+C_{2} e^{-k x}
\end{array}\right.
$$

where $k=\frac{\sqrt{c^{4}-4 \lambda^{2}}}{2 c}$. Due to known spectral properties of 1D Dirac operator, it is clear that if one finds a non-zero solution corresponding to $\lambda$,

$$
\lambda \in\left(-\frac{c^{2}}{2}, \frac{c^{2}}{2}\right)
$$

then such $\lambda$ belongs to the point spectrum of the operator. Using (2.0), (2.2), (3.1), one can express $C_{1,2}^{(3)}$ and $C_{1,2}^{(4)}$ through the $C_{1,2}^{(1)}$ and $C_{1,2}^{(2)}$ :

$$
\begin{aligned}
& \left(\begin{array}{l}
C_{1}^{(3)} \\
C_{2}^{(3)}
\end{array}\right)=\left(\begin{array}{l}
C_{1}^{(4)} \\
C_{2}^{(4)}
\end{array}\right)=\left(\begin{array}{cc}
\gamma-1 & -\gamma-1 \\
\gamma & -\gamma
\end{array}\right)\left(\begin{array}{l}
C_{1}^{(1)} \\
C_{2}^{(1)}
\end{array}\right)= \\
& T\left(\begin{array}{l}
C_{1}^{(1)} \\
C_{2}^{(1)}
\end{array}\right), \quad\left(\begin{array}{l}
C_{1}^{(1)} \\
C_{2}^{(1)}
\end{array}\right)=\left(\begin{array}{l}
C_{1}^{(2)} \\
C_{2}^{(2)}
\end{array}\right) .
\end{aligned}
$$

where $\gamma=\frac{\theta}{4}=\frac{\alpha}{4 c} \sqrt{\frac{c^{2}+2 \lambda}{c^{2}-2 \lambda}}$. The reduced (i.e. $2 \times 2-$ matrix $T$ due to symmetry) transfer-matrix is as follows:

It is simple to find the eigenvalues $\mu_{1,2}$ and the eigenvectors $e_{1,2}$ of the transfer-matrix $T$ :

$$
\begin{gathered}
\mu_{1,2}=\frac{-1 \pm \sqrt{1-8 \gamma}}{2}, e_{1,2}=\left(\begin{array}{l}
a_{1,2} \\
b_{1,2}
\end{array}\right) \\
\frac{a_{1,2}}{b_{1,2}}=\frac{2 \gamma-1 \pm \sqrt{1-8 \gamma}}{2 \gamma} .
\end{gathered}
$$

Relation (3.3) gives one the ratio of the coefficients $C_{1}^{(1)}$ and $C_{2}^{(1)}$ :

$$
\frac{C_{1}^{(1)}}{C_{2}^{(1)}}=\frac{2 \gamma-1 \pm \sqrt{1-8 \gamma}}{2 \gamma}
$$

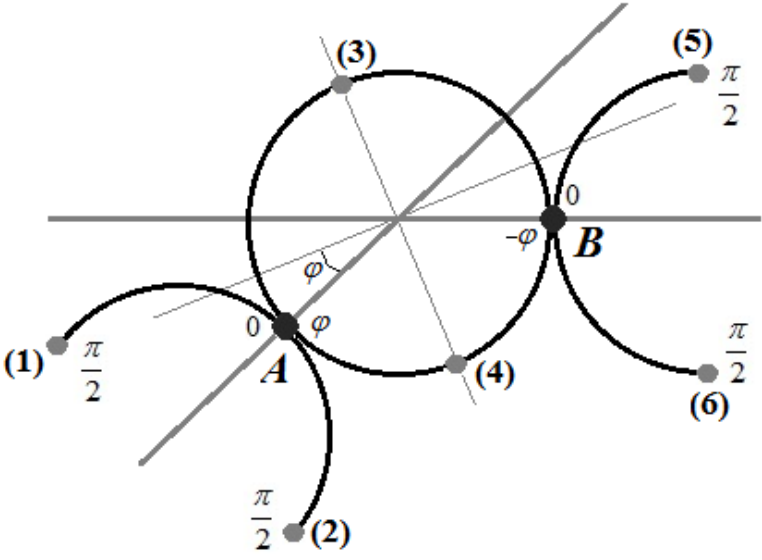

Fig. 4. The central region of the graph. $\varphi$ is the angular deviation of the coupling point from the diameter.

It is well known (see, e.g., [21]) that real $\lambda$ belongs to the continuous spectrum of the Dirac operator for semi-infinite chain if $\left|\mu_{1,2}\right|=1$. In contrast, if $\left|\mu_{1}\right|<1$ (or $\left|\mu_{2}\right|<1$ ) then the corresponding $\lambda$ belongs to the point spectrum of the Dirac operator for semi-infinite chain (one obtains square integrable solution at the chain). In our case, it means that $8 \gamma<1$ (the corresponding eigenvector of the transfer-matrix gives one the initial conditions for this solution). Keeping in mind this observation, we will consider the central region of the graph - chains coupling.

This part of the graph is shown in Fig. 4. There is only one difference between the central circle and any other circle of the chain - the coupling points A and B do not belong to the same diameter.

The system of equations for values of $\psi_{1,2}^{(1,2)}, \psi_{1,2}^{(3,4)}$ and $\psi_{1,2}^{(5,6)}$ consists of two parts corresponding to A and $\mathrm{B}$ respectively. It looks like (2.2), but the values of the functions $\psi_{1,2}^{(3,4)}$ are calculated at $\varphi$ (for $\mathrm{A}$ ) and $-\varphi$ (for B), i.e.:

$$
\begin{gathered}
\left\{\begin{array}{l}
\psi_{2}^{(1)}(0)+\psi_{2}^{(2)}(0)+\psi_{2}^{(3)}(\varphi)+\psi_{2}^{(4)}(\varphi)=-\frac{i \alpha}{c} \psi_{1}^{(1)}(0), \\
\psi_{1}^{(1)}(0)=\psi_{1}^{(2)}(0)=\psi_{1}^{(3)}(\varphi)=\psi_{1}^{(4)}(\varphi) .
\end{array}\right. \\
\left\{\begin{array}{l}
C_{1}^{(1)}=C_{1}^{(2)} \\
C_{2}^{(1)}=C_{2}^{(2)}
\end{array}, \quad \begin{array}{l}
C_{1}^{(5)}=C_{1}^{(6)}, \\
C_{2}^{(5)}=C_{2}^{(6)} .
\end{array}\right. \\
\left\{\begin{array}{l}
\psi_{2}^{(3)}(-\varphi)+\psi_{2}^{(4)}(-\varphi)+\psi_{2}^{(5)}(0)+\psi_{2}^{(6)}(0)=-\frac{i \alpha}{c} \psi_{1}^{(5)}(0) \\
\psi_{1}^{(3)}(-\varphi)=\psi_{1}^{(4)}(-\varphi)=\psi_{1}^{(5)}(0)=\psi_{1}^{(6)}(0) .
\end{array}\right.
\end{gathered}
$$

Here we take into account the symmetry. Substituting the explicit expressions for functions $\psi$, one expresses the coefficients $C_{1,2}^{(5)}, C_{1,2}^{(6)}$ in terms of $C_{1,2}^{(1)}, C_{1,2}^{(2)}$ : 


$$
\begin{aligned}
& \left(\begin{array}{l}
C_{1}^{(5)} \\
C_{2}^{(5)}
\end{array}\right)=\left(\begin{array}{ll}
a_{11} & a_{12} \\
a_{21} & a_{22}
\end{array}\right)\left(\begin{array}{l}
C_{1}^{(1)} \\
C_{2}^{(1)}
\end{array}\right) \\
& a_{11}=e^{-2 k \varphi}+\frac{\theta^{2}}{16}\left(1-e^{-4 k \varphi}\right), \\
& a_{12}=-\frac{\theta}{4}\left(e^{k \varphi}-e^{-3 k \varphi}\right)-\frac{\theta^{2}}{16}\left(1-e^{-4 k \varphi}\right), \\
& a_{21}=-\frac{\theta}{4}\left(e^{k \varphi}-e^{-3 k \varphi}\right)+\frac{\theta^{2}}{16}\left(1-e^{-4 k \varphi}\right), \\
& a_{22}=e^{2 k \varphi}-\frac{\theta^{2}}{16}\left(1-e^{-4 k \varphi}\right) .
\end{aligned}
$$

Using Eq. (3.4), (3.5), one obtains the relation between $\theta=\theta(\lambda)=\frac{\alpha}{c} \sqrt{\frac{c^{2}+2 \lambda}{c^{2}-2 \lambda}}, k=k(\lambda)=\frac{\sqrt{c^{4}-4 \lambda^{2}}}{2 c}$.

The result is as follows:

$$
\begin{gathered}
\theta=-2 e^{2 k \varphi}-2 e^{k \varphi} . \\
\theta=3 e^{k \varphi}-e^{2 k \varphi}+\sqrt{e^{2 k \varphi}-6 e^{3 k \varphi}+e^{4 k \varphi}} \\
\theta=3 e^{k \varphi}-e^{2 k \varphi}-\sqrt{e^{2 k \varphi}-6 e^{3 k \varphi}+e^{4 k \varphi}} .
\end{gathered}
$$

Equations (3.6), (3.7) and (3.8) are equations for $\lambda$. To be an eigenvalue of the Dirac operator for the bent chain, $\lambda$ should be a root of at least one of these equation. To complete the proof of the main theorem, we consider the following two lemmas:

Lemma 1. Equation (3.6) have at least one solution if and only if $\alpha<0$.

Lemma 2. Equations (3.7) and (3.8), in common, have at least one solution if and only if $\alpha<0$ and

$$
\varphi \geq \frac{2 \ln (3+2 \sqrt{2})(r+1)}{c \sqrt{2 r^{2}+2}}, \quad r=\left(\frac{c}{\alpha}(6 \sqrt{2}+8)\right)^{2} .
$$

Equation (3.7) have at least two another solutions if and only if $\alpha<0$ and

$$
\varphi \in\left[\begin{array}{l}
{\left[w_{0} ; w_{1}\right) \cup\left(w_{2} ;+\infty\right), \quad \alpha \geq-4 c} \\
{\left[w_{0} ; w_{2}\right), \quad \alpha<-4 c}
\end{array}\right.
$$

where

$$
\begin{aligned}
& w_{1,2}=\frac{2}{c} \ln \left(\frac{3 \alpha \pm \alpha \sqrt{1-\frac{2 \alpha}{c}}}{2(4 c+\alpha)}\right), \\
& w_{0}=\frac{2}{c} \ln \left(\frac{3 \sqrt{c}+\sqrt{9 c-4 \alpha}}{2 \sqrt{c}}\right) .
\end{aligned}
$$

The lemmas are proved by means of analyzing the behavior of functions including in the equations. Summarizing the results of Lemma 1 and Lemma 2, we complete the proof of the main theorem.

\section{Concluding remarks}

The paper deals with the spectrum description of the Dirac operator for a quantum graph having the form of bent chain of rings. Such models can be applied to the description of electron behavior in grapheme-based nanostructures like grapheme nanoribbon (see, e.g., [23]). The main result of the paper is the description of the conditions ensuring the existence of bound states. As for the continuous spectrum for the bending chain, it is not so interesting because it coincides with that for the corresponding straight infinite chain (finite rank perturbation cannot lead to a change of the continuous spectrum). It is interesting to compare the result with the corresponding theorem concerning to the analogous quantum graph with the Schrodinger operator (i.e. nonrelativistic particle) [8]. In the both cases, bound states appear only if there is negative delta-coupling at the graph vertices. From the physical point of view deltacoupling is related to physical characteristics of the rings connections. For non-relativistic particle, this statement was proved for more complicated cases of branched graphs too $[9,10]$. Naturally, the conditions depend on the bending angle. For the relativistic case, i.e. for the Dirac operator, one can use the transfer-matrix approach analogously to the non-relativistic case (for the Schrodinger operator).

This work was partially financially supported by the Government of the Russian Federation (grant 074-U01), by the Ministry of Education and Science of the Russian Federation (GOSZADANIE 2014/190, Projects No 14.Z50.31.0031 and No. 1.754.2014/K)), by grants MK-5001.2015.1 and MK5161.2016.1 of the President of the Russian Federation, by grant 16-11-10330 of Russian Science Foundation.

\section{References}

1. D. Wei, Y. Liu, Advanced Materials, 20, 28152841, (2008)

2. I.S. Lobanov, I.Yu. Popov, Nanosystems: Physics, Chemistry, Mathematics, 3(2), 6-28 (2012)

3. E.L. Korotyaev, K.M. Schmidt, L. Reine Angew. Math., 670, 217-248 (2012)

4. H. Santos, A. Ayuela, W. Jaskolski, M. Pelc, L. Chico., Phys. Rev. B., 80, 035436 (2009)

5. G. Berkolaiko, P Kuchment, Introduction to Quantum Graphs, Mathematical Surveys and Monographs 186; 270 pp; AMS (2013), Providence, RI

6. P. Exner, J.P. Keating, P. Kuchment, T. Sunada, A. Teplyaev, S. Benvegn, L. Dabrowski (eds.), Analysis on Graphs and Its Applications. Proc. Symp. Pure Math. 77 (Amer. Math.Soc., Providence, RI, 2008)

7. O. Post. Spectral Analysis on Graph-like Spaces. (Berlin, Springer, 2012)

8. P. Duclos, P. Exner, O. Turek, J. Phys. A: Math. Theor, 41, 415206/1-18 (2008)

9. I.Yu. Popov, P.I. Smirnov, Physics Letters A, 377(6), 439-442 (2013) 
10. I.Yu. Popov, A.N. Skorynina, I.V. Blinova, J. Math. Phys., 55(3), 033504/1-19 (2014)

11. S. Albeverio, F. Gesztesy, R. Høegh-Krohn, H. Holden, Solvable models in quantum mechanics, 2nd ed., with an appendix by Pavel Exner, AMS (Chelsea, Providence, RI, 2005)

12. S. Albeverio and P. Kurasov, Singular perturbations of differential operators, London Math. Soc. Lecture Note Ser., 271, (Cambridge Univ. Press, Cambridge, 2000)

13. B.S. Pavlov, Uspekhi Mat. Nauk., 42(6), 99-131 (1987)

14. S. Clark, F. Gesztesy. Trans. Am. Math. Soc., 358, 3475 (2002).

15. W.B. Huddell III, R.J Hughes. J. Phys. A: Math. Gen., 38(22), 4803 , (2005).

16. S. Benvegnù. Letters in Math. Phys. 30(2):159167.(1994)

17. F. Gesztesy, P. Seba, Lett. Math. Phys. 13, 345-358 (1987)

18. K.Yoshitomi. Michigan Math. J. 2009, 58, 363-384

19. R.J. Hughes, Integral Equations Operator Theory, 31, 436-448 (1998)

20. P. Šeba, Lett. Math. Phys., 18, 77-86 (1989)

21. E.N.Grishanov, D.A.Eremin, D.A.Ivanov, I.Y.Popov, Chin. Phys. B, 25 (4), 047303 (2016)

22. B.M. Levitan, I.S. Sargsyan. Sturm-Liouville and Dirac Operators (Moscow, Nauka, 1988)

23. K.Wakabayashi, K.Sasaki, T.Nakanishi, T.Enoki, Sci. Technol. Adv. Mater., 11, 054504 (18 pp) (2010) 